Pacific Journal of Mathematic

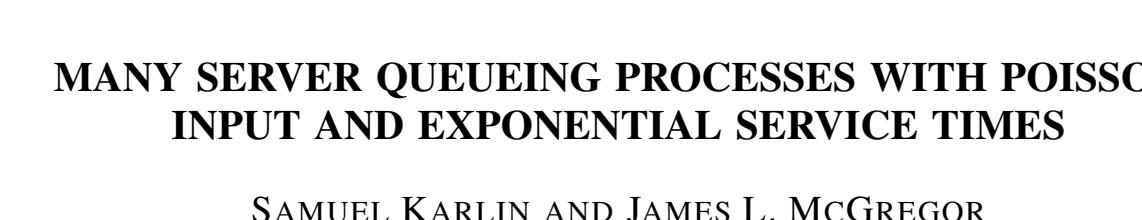




\section{MANY SERVER QUEUEING PROCESSES WITH \\ POISSON INPUT AND EXPONENTIAL \\ SERVICE TIMES}

\section{Samuel Karlin and James McGregor}

1. Introduction. A birth and death process is a stationary Markoff process whose state space is the non-negative integers and whose transition probability matrix

$$
P_{i j}(t)=\operatorname{Pr}\{x(t)=j \mid x(0)=i\}
$$

satisfies the conditions (as $t \rightarrow 0$ )

$$
p_{i j}(t)= \begin{cases}\lambda_{i} t+o(t) & \text { if } j=i+1, \\ \mu_{i} t+o(t) & \text { if } j=i-1, \\ 1-\left(\lambda_{i}+\mu_{i}\right) t+o(t) & \text { if } j=i,\end{cases}
$$

where $\lambda_{i}>0$ for $i \geqq 0, \mu_{i}>0$ for $i \geqq 1$, and $\mu_{0} \geqq 0$. The process is called a queueing process if $\mu_{0}=0$ and $\lambda_{i}=\lambda$ for all $i$. The state of the system is then interpreted as the length of a queue for which the inter-arrival times have a negative exponential distribution with parameter $\lambda$, and for which the service times have a negative exponential distribution whose parameter $\mu_{n}$ depends on the length of the line. The classical case of a single server queue corresponds to $\mu_{n}=\mu, n \geqq 1$, and has been discussed by Reuter and Lederman [9] and Bailey [1].

The so-called telephone trunking problem (Feller [3]) arises from a queueing process with infinitely many servers, each of whose service time distribution has the same parameter $\mu$, so that $\mu_{n}=n \mu, n \geqq 1$. Besides these two special cases, we discuss a queue with $n$ servers, each of whose service time has a negative exponential distribution with the same parameter $\mu$, so that $\mu_{k}=k \mu$ for $1 \leqq k \leqq n, \mu_{k}=n \mu$ for $k \geqq n$. Our methods can also be used to study queueing processes with several servers whose service times have negative exponential distributions not all with the same parameter.

A sample of the type of problems treated is as follows :

(1) to obtain a usable formula for the transition probability $P_{i j}(t)$;

(2) to compute the distribution of the length of a busy period;

(3) to compute the distribution of the number of customers served during a busy period;

(4) to compute the distribution of the maximum length of the queue during a busy period; and similar questions.

Received May 29, 1957. 
At this point it would be of some interest to tie the investigations of this paper together with the other work in this field. It is important to emphasize that we are concerned primarily with the analysis of non-stationary problems associated with the $n$ server queuing process. The equilibrium distribution of length of line for the case of exponential service time and Poisson input is trivial to determine. The equilibrium situation for the general input process with exponential service time and $n$ servers was completely resolved by Kendall [7] who, in addition, evaluated explicitly the distribution of waiting time for a randomly arriving customer. A non-constructive existence theorem for the stationary distribution of a general input process and a general service time distribution was given in [8]. In contrast, a considerable amount of insight regarding transient behavior has been attained in the case of the one server queue. For an elegant treatment of this case the reader is referred to the work of Takács [10].

Part of the significance in resolving the problems related to the $n$ server queue even subject to the special assumptions of exponential service time and Poisson input, in addition to its independent interest, rests on the following two observations :

(1) the general queueing process with the corresponding appropriate parameters behaves on an average like the exponential case, and

(2) the solution for the exponential case may be suggestive as to the nature of the answers in the general case.

Our detailed analysis regarding queueing processes with exponential service time, Poisson input, and many servers derives from our knowledge of the refined structure of birth and death processes developed in [4] and [5]. We rely primarily on the theory of recurrence and absorption for a birth and death process as spelled out in [5].

In this connection, although the parameter $\mu_{0}$ is zero for a queueing process it is convenient to consider, along with a queueing process, related birth and death processes for which $\mu_{0}$ is positive. Such a process has an ignored absorbing state at -1 , a state in which the system remains forever once it arrives there. When the system is in the zero state and a transition occurs, the system moves to state 1 with probability $\lambda_{0} /\left(\lambda_{0}+\mu_{0}\right)$ and is absorbed with probability $\mu_{0} / \lambda_{0}+\mu_{0}$.

The infinitesimal matrix of the general birth and death process is of the form

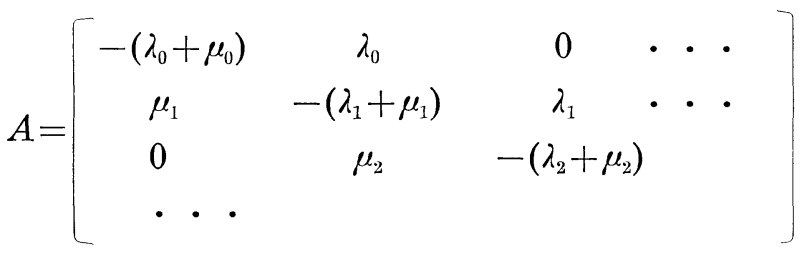

This matrix determines a system of polynomials by means of the re- 
cursion relations

$$
\left\{\begin{aligned}
Q_{0}(x) & =1, \\
-x Q_{0}(x) & =-\left(\lambda_{0}+\mu_{0}\right) Q_{0}(x)+\lambda_{0} Q_{1}(x), \\
-x Q_{n}(x) & =\mu_{n} Q_{n-1}(x)-\left(\lambda_{n}+\mu_{n}\right) Q_{n}(x)+\lambda_{n} Q_{n+1}(x) .
\end{aligned}\right.
$$

It is shown in [4] that there is a positive regular measure $\psi$ on $0 \leqq x<\infty$ for which the orthogonality relations

$$
\int_{0}^{\infty} Q_{i}(x) Q_{j}(x) d \psi(x)=\frac{\delta_{i j}}{\pi_{j}} \quad i, j=0,1, \cdots
$$

where $\pi_{0}=1, \pi_{n}=\frac{\lambda_{0} \lambda_{1} \cdots \lambda_{n-1}}{\mu_{1} \mu_{2} \cdots \mu_{n}}$, are valid. In the case of a queueing process, the measure $\psi$ is unique [4], and moreover the transition probability matrix $P(t)=\left(P_{i j}(t)\right)$ of the process is uniquely determined by $A$. It has the representation

$$
P_{i j}(t)=\pi_{j} \int_{0}^{\infty} e^{-x t} Q_{i}(x) Q_{j}(x) d \psi(x) .
$$

This is an extremely useful form of expression for the transition probability function, and our first task will be to compute the polynomials $\left\{Q_{n}(x)\right\}$ and the spectral measure $\psi$ belonging to the various queueing processes.

This is accomplished in the following section based on a formula which connects the Stieltjes transform of the spectral measure of the process and the Stieltjes transform of the spectral measure of the associated process. Once the Stieltjes transform of the spectral measure is known, then recourse to the classical inversion formulas of Stieltjes transforms enables us to determine the spectral measure itself. This is done in $\S 4$. Previous to that in $\S 3$ a discussion of the infinite server queueing process is made. Here we recognize the corresponding polynomials as the classical Poisson-Charlier polynomials which are known to be orthogonal with respect to an appropriate Poisson distribution. Some remarks are appended describing the nature of first passage distributions of the states of the system in this case.

In the following section the spectral measure and the polynomials of the $n$ server queueing process are explicitly determined. The polynomials are found to be expressible as combinations of the familiar Chebycheff polynomials of the first and second kind and Poisson-Charlier polynomials.

In $\S 5$ the previous theory is specialized to the one and two server process. Further detailed information regarding these processes is collected. 
$\S 6$ is devoted to a complete study of various probability distributions associated with queueing problems of one and two servers. The transition probabilities of the Markov process describing the waiting line are explicitly determined. The distribution to the length of the busy period, the distribution of the number of customers served during a busy period, and other such distributions are exhibited. In the following section the corresponding results for the $n$ server queue are written out. The proofs of these assertions for the general case, exceedingly more complicated in detail, are carried out in the discussion of Appendix A. In $\S 8$ we derive the distribution of the maximum length of line during a busy period. The second appendix summarizes the properties of a new system of polynomials related to the Poisson-Charlier polynomials.

2. The related processes. From a given birth and death process with infinitesimal matrix (1.3) a new process is obtained by stopping the given process whenever the state 0 is reached. For this new process the state 0 is an absorbing state, and if we ignore this state the process is a birth and death process for which the parameter $\mu_{0}$ is positive. The waiting time in any state $i \geqq 1$ has the same distribution for both the original and the new process, and moreover both processes have the same post exit distributions for each state $i \geqq 1$. Consequently the infinitesimal matrix of the new process (with the state 0 ignored) is

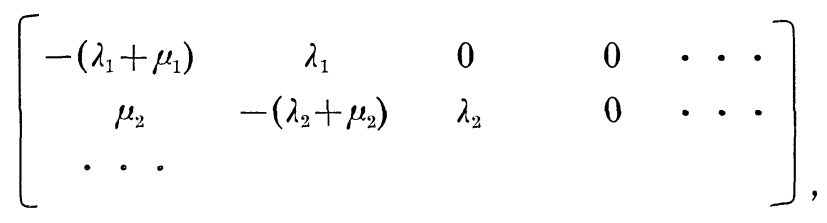

which is obtained from (1.3) by removing the zero row and zero column. The polynomials defined by

$$
\left\{\begin{array}{l}
Q_{0}^{(0)}(x)=0, Q_{1}^{(0)}(x)=-\frac{1}{\lambda_{0}}, \\
-x Q_{n}^{(0)}(x)=\mu_{n} Q_{n-1}^{(0)}(x)-\left(\lambda_{n}+\mu_{n}\right) Q_{n}^{(0)}(x)+\lambda_{n} Q_{n+1}^{(0)}(x), n \geqq 1
\end{array}\right.
$$

are called the associated polynomials of the system $\left\{Q_{n}(x)\right\}$. It is seen that, except for the constant factor $-\frac{1}{\lambda_{0}}$, they are the polynomials belonging to the new birth and death process. Consequently the transition probability matrix $\left(\tilde{P}_{i j}(t)\right), i, j \geqq 1$, of the new process is given by

$$
\tilde{P}_{i j}(t)=\frac{\mu_{1}}{\lambda_{0}} \pi_{j} \int_{0}^{\infty} e^{-x t}\left[-\lambda_{0} Q_{i}^{(0)}(x)\right]\left[-\lambda_{0} Q_{j}^{(0)}(x)\right] d \alpha(x)
$$


where $\alpha$ is the spectral measure of the new process. In $[5, \S 8]$ it is shown that the Stieltjes transforms of the spectral measures $\psi$ and $\alpha$ of the two processes,

$$
B(s)=\int_{0}^{\infty} \frac{d \psi}{x-s}, \quad C(s)=\int_{0}^{\infty} \frac{d \alpha}{x-s},
$$

are related by the identity

$$
B(s)=\frac{1}{\lambda_{0}+\mu_{0}-s-\lambda_{0} \mu_{1} C(s)} .
$$

This identity is the basic tool used in calculating the spectral measure of the $n$ server queueing process. Once the function $B(s)$ has been found the measure can be computed by means of known formulas for inverting the Stieltjes transform. See [5] for a discussion of this inversion relative to the identity (2.5), and [12], [11] for the general inversion problem.

By iterating (2.5) a relation will be obtained between the spectral measure of the original process and the spectral measure of the process obtained from the original one by stopping it whenever the state $n$ is reached. Denote the spectral measure of the original process by $\psi_{0}$, and the spectral measure of the process obtained from the original one by stopping it whenever the $n$th state is reached, by $\psi_{n+1}$. Then if

$$
B_{k}(s)=\int_{0}^{\infty} \frac{d \psi_{k}(x)}{x-s}
$$

(2.5) gives

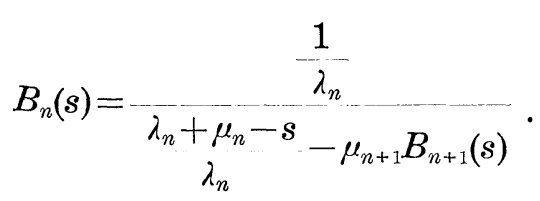

It is clear that

$$
B_{0}(s)=\frac{\alpha_{n} B_{n}(s)+\beta_{n}}{\gamma_{n} B_{n}(s)+\delta_{n}}
$$

where $\alpha_{n}, \beta_{n}, \gamma_{n}, \delta_{n}$ are (not uniquely determined) functions of $s$. Permissible choices for $n=0,1$ are $\alpha_{0}=1, \beta_{0}=\gamma_{0}=0, \delta_{0}=1$ and $\alpha_{1}=0, \beta_{1}=1 / \lambda_{0}$, $\gamma_{1}=-\mu_{1}, \delta_{1}=\left(\lambda_{0}+\mu_{0}-s\right) / \lambda_{0}=Q_{1}(s)$. Substituting (2.7) into (2.8) it is found that the coefficient functions can be determined by the relations

$$
\begin{gathered}
\alpha_{n+1}=-\mu_{n+1} \beta_{n}, \\
\lambda_{n} \beta_{n+1}=\alpha_{n}+\left(\lambda_{n}+\mu_{n}-s\right) \beta_{n},
\end{gathered}
$$




$$
\begin{gathered}
\gamma_{n+1}=-\mu_{n+1} \delta_{n} \\
\lambda_{n} \delta_{n+1}=\gamma_{n}+\left(\lambda_{n}+\mu_{n}-s\right) \delta_{n}
\end{gathered}
$$

and hence

$$
B_{0}(s)=-\frac{\mu_{n} Q_{n-1}^{(0)}(s) B_{n}(s)-Q_{n}^{(0)}(s)}{\mu_{n} Q_{n-1}(s) B_{n}(s)-Q_{n}(s)}
$$

3. The queue with infinitely many servers. The polynomials belonging to this process will be denoted by $p_{n}(x)=p_{n}(x, \lambda, \mu)$. They satify

$$
\left\{\begin{array}{l}
p_{0}(x)=1, \\
-x p_{0}(x)=-\lambda p_{0}(x)+\lambda p_{1}(x), \\
-x p_{n}(x)=n \mu p_{n-1}(x)-(\lambda+n \mu) p_{n}(x)+\lambda p_{n+1}(x), \quad n \geqq 1 .
\end{array}\right.
$$

They can be identified in terms of the Poisson-Charlier polynomials $c_{n}(x, a),[2$, Vol. 2, p. 226], which satisfy

$$
\left\{\begin{array}{l}
c_{0}(x, a)=1, \\
-x c_{0}(x, a)=-a c_{0}(x, a)+a c_{1}(x, a), \\
-x c_{n}(x, a)=n c_{n-1}(x, a)-(n+a) c_{n}(x, a)+a c_{n+1}(x, a), \quad n \geqq 1
\end{array}\right.
$$

Thus

$$
p_{n}(x, \lambda, \mu)=c_{n}\left(\frac{x}{\mu}, \frac{\lambda}{\mu}\right),
$$

The measure with respect to which the Poisson-Charlier polynomials are orthogonal consists of masses

$$
j(x)=e^{-a} \frac{a^{x}}{x !} \text { at } x=0,1,2, \cdots
$$

Hence the spectral measure $\psi$ of the infinite server queue consists of masses

$$
d \psi(x)=\frac{e^{-a} a^{n}}{n !} \text { at } x_{n}=n \mu, n=0,1, \cdots
$$

where $a=\frac{\lambda}{\mu}$. From well-known properties of the Poisson-Charlier polynomials [2] it is found that

$$
p_{n}(k \mu)=p_{k}(n \mu)
$$




$$
\sum_{n=0}^{\infty} p_{n}(\mu x) \frac{z^{n}}{n !}=e^{z}\left(1-\frac{z}{a}\right)^{x}, \quad a=\frac{\lambda}{\mu} .
$$

The representation of the transition probability matrix is

$$
\begin{aligned}
P_{n k}(t) & =\pi_{k} \int_{0}^{\infty} e^{-x t} p_{n}(x) p_{k}(x) d \psi(x) \\
& =\frac{a^{k}}{k !} \sum_{r=0}^{\infty} e^{-r \mu t} p_{n}(r \mu) p_{k}(r \mu) e^{-a} \frac{a^{r}}{r !} .
\end{aligned}
$$

In particular

$$
\begin{aligned}
P_{n 0}(t) & =e^{-a} \sum_{r=0}^{\infty} p_{r}(n \mu) \frac{\left(a e^{-\mu t}\right)^{r}}{r !} \\
& =e^{-a\left(1-e^{-\mu t}\right)}\left(1-e^{-\mu t}\right)^{n},
\end{aligned}
$$

and

$$
\begin{aligned}
\sum_{k=0}^{\infty} P_{n k}(t) z^{k} & =e^{-a} \sum_{r=0}^{\infty} p_{r}(n \mu) \frac{\left(a e^{-\mu t}\right)^{r}}{r !} \sum_{k=0}^{\infty} p_{k}(r \mu) \frac{(\alpha z)^{k}}{k !} \\
& =e^{-a(1-z)\left(1-e^{-\mu t}\right)}\left[1-(1-z) e^{-\mu t}\right]^{n} .
\end{aligned}
$$

The last two formulas are well-known and can be found by generating function techniques [3, p. 396].

Now consider the spectral measure $\alpha$ of the process obtained by stopping the infinitely many server process when the zero state is reached. Writing (2.5) in the form

$$
C(s)=\int_{0}^{\infty} \frac{d \alpha(x)}{x-s}=\frac{1}{\lambda \mu}\left[\lambda-s-\frac{1}{\int_{0}^{\infty} \frac{d \psi(x)}{x-s}}\right]
$$

and noting that

$$
B(s)=\int_{0}^{\infty} \frac{d \psi(x)}{x-s}=e^{-a} \sum_{n=0}^{\infty} \frac{a^{n}}{n !(n \mu-s)},
$$

we see that $C(s)$ is a metomorphic function whose poles are simple poles at the zeros of $B(s)$. Thus $\alpha$ is a discrete distribution whose masses are located at the zeros of $B(s)$. The zeros $s_{0}<s_{1}<s_{2}<\cdots$ of $B(s)$ are all simple, $n \mu<s_{n}<(n+1) \mu$, and the mass $\alpha_{n}$ of the distribution $\alpha$ which is located at $s_{n}$ is

$$
\alpha_{n}=\frac{+1}{\lambda \mu B^{\prime}\left(s_{n}\right)} .
$$


(See [5] for a more complete discussion. The function here denoted by $B(s)$ is there denoted by $B(-s)$ ). For many purposes it is sufficient to know $s_{n}$ and $\alpha_{n}$ for only the first few values of $n$. For example the first passage time distribution

$$
F_{10}(t)=\operatorname{Pr}\{X(\tau)=0 \text { for some } \tau, 0<\tau \leqq t \mid X(0)=1\}
$$

of the original process is

$$
\begin{aligned}
F_{10}(t) & =\mu \int_{0}^{\infty} \frac{1-e^{-x t}}{x} d \alpha(x) \\
& =1-\mu \sum_{n=0}^{\infty} \frac{\alpha_{n}}{s_{n}} e^{-s_{n} t}
\end{aligned}
$$

and for large $t$ only the first few terms are important.

For purposes of numerical computation the following facts, stated for the case $\mu=1$, are useful:

(i) for all $a>0, \frac{d s_{n}}{d a}<0$, and

(ii) $s_{n}<s_{n+1}-1$.

To prove (i) it is noted that

$$
\sum_{k=0}^{\infty} \frac{a^{k}}{k !\left[k-s_{n}(a)\right]} \equiv 0, \quad a>0,
$$

and hence

$$
\sum_{k=0}^{\infty} \frac{k a^{k-1}}{k !\left[k-s_{n}(a)\right]}+\sum_{k=0}^{\infty} \frac{a^{k}}{k !\left[k-s_{n}(a)\right]^{2}}\left(\frac{d s_{n}}{d a}\right)=0 .
$$

Consequently it is sufficient to show that

$$
\sum_{k=0}^{\infty} \frac{k a^{k}}{k !\left(k-s_{n}\right)}>0
$$

Now $n<s_{n}<n+1$ so

$$
\sum_{k=n}^{\infty} \frac{a^{k}}{k !\left(k-s_{n}\right)}=-\sum_{k=0}^{n-1} \frac{a^{k}}{k !\left(k-s_{n}\right)}>0
$$

and

$$
\sum_{k=0}^{\infty} \frac{k a^{k}}{k !\left(k-s_{n}\right)}=\sum_{k=1}^{n} \sum_{r=k}^{\infty} \frac{a^{r}}{r !\left(r-s_{n}\right)}+\sum_{k=n+1}^{\infty} \frac{(k-n) a^{n}}{k !\left(k-s_{n}\right)}>0 .
$$

To prove (ii) it is observed that 


$$
B(s, a)=e^{-a} \sum_{k=0}^{\infty} \frac{a^{k}}{k !(k-s)}
$$

satisfies the functional equation

$$
\frac{\partial B(s, a)}{\partial a}=-[B(s, a)-B(s-1, a)] .
$$

Because of (i) and (3.14) $\left.\frac{\partial B(s, a)}{\partial a}\right|_{s=s_{n}}>0$ which, together with (3.15), gives $B\left(s_{n}-1, a\right)>B\left(s_{n}, a\right)=0$. Now $B(s, a)=B(s)$ is monotone increasing in each interval $n<s<n+1, n=0,1,2, \cdots$. Consequently

$$
s_{n-1}<s_{n}-1 \text {. }
$$

The following table gives $s_{n}$ and $B^{\prime}\left(s_{n}\right)$ for $n=0,1,2$ and several values of $a$.

\begin{tabular}{|c|rrrrrr|}
\hline $\mathrm{a}$ & $\begin{array}{c}\hat{\mathrm{s}}_{1} \\
\text { First root }\end{array}$ & $\begin{array}{c}\mathrm{B}^{\prime}\left(\hat{\mathrm{s}}_{1}\right) \\
\text { At first root }\end{array}$ & $\begin{array}{c}\hat{\mathrm{s}}_{2} \\
\text { Second root }\end{array}$ & $\mathrm{B}^{\prime}\left(\hat{\mathrm{s}}_{2}\right)$ & $\hat{\mathrm{s}}_{3}$ & $\mathrm{~B}^{\prime}\left(\hat{\mathrm{s}}_{3}\right)$ \\
.5 & .65116 & 6.54006 & 1.88388 & 10.21023 & 2.97092 & 25.00957 \\
1.0 & .45027 & 8.47902 & 1.72376 & 8.90911 & 2.88131 & 12.91379 \\
1.5 & .31745 & 13.63762 & 1.58297 & 11.60410 & 2.77136 & 13.41379 \\
2.0 & .22517 & 23.92535 & 1.46574 & 17.38949 & 2.66252 & 17.55924 \\
\hline
\end{tabular}

4. The spectral measure and the polynomials of the $n$ server queue. For the $n$ server process

$$
\left\{\begin{array}{l}
\lambda_{k}=\lambda, \\
\mu_{k}= \begin{cases}k \mu, & k \leqq n, \\
n \mu, & k \geqq n .\end{cases}
\end{array}\right.
$$

Hence

$$
Q_{k}(x)=p_{k}(x, \lambda, \mu), \quad k \leqq n,
$$

where $p_{n}$ is given by (3.3). The polynomials for $k \geqq n$ will be determined presently. As in $\S 2$ we denote the spectral measure of the process by $\psi_{0}$ and the spectral measure of the process obtained if the given process is stopped whenever the state $k(k \geqq 0)$ is reached by $\psi_{k+1}$. If

$$
B_{k}(s)=\int_{0}^{\infty} \frac{d \psi_{k}(x)}{x-s}
$$

then from (2.9)

$$
B_{0}(s)=-\frac{n \mu Q_{n-1}^{(0)}(s) B_{n}(s)-Q_{n}^{(0)}(s)}{n \mu Q_{n-1}(s) B_{n}(s)-Q_{n}(s)} .
$$


Because of (4.1), $B_{n}(s)=B_{n+1}(s)$ and hence (2.7) gives

$$
B_{n}(s)=\frac{\lambda+n \mu-s-V(\lambda+n \mu-s)^{2}-4 n \lambda \mu}{2 n \lambda \mu},
$$

where, in accordance with (4.3), the square root is taken positive for $s<0$. Substituting (4.5) into (4.4) and rationalizing we obtain, with the use of the identity $\lambda_{n-1} \pi_{n-1}\left[Q_{n} Q_{n-1}^{(0)}-Q_{n}^{(0)} Q_{n-1}\right]=1$, where $\lambda_{n} \pi_{n}=\lambda\left(\frac{\lambda}{\mu}\right)^{n} \frac{1}{n !}$,

$$
B_{0}(s)=-\frac{L_{n}(s)}{K_{n}(s)}
$$

where

$$
\begin{aligned}
L_{n}(s)= & 4 \lambda^{2} Q_{n}(s) Q_{n}^{(0)}(s)+4 n \lambda \mu Q_{n-1}(s) Q_{n-1}^{(0)}(s) \\
& -2 \lambda(\lambda+n \mu-s)\left[Q_{n}(s) Q_{n-1}^{(0)}(s)+Q_{n}^{(0)}(s) Q_{n-1}(s)\right] \\
& +2(n-1) !\left(\begin{array}{c}
\mu \\
\lambda
\end{array}\right)^{n-1} \sqrt{(\lambda+n \mu-s)^{2}-4 n \lambda \mu} \\
K_{n}(s)= & 4 \lambda^{2}\left[Q_{n}^{2}(s)-Q_{n-1}(s) Q_{n+1}(s)\right] \\
= & 4 n \lambda \mu\left[Q_{n-1}^{2}(s)-Q_{n}(s) Q_{n-2}(s)\right]-4 \lambda \mu Q_{n}(s)\left[Q_{n-1}(s)-Q_{n-2}(s)\right] . \\
L_{n}(s)= & 4 \lambda^{2}\left[Q_{n}(s) Q_{n}^{0}(s)-\frac{1}{2}\left\{Q_{n-1}^{(0)}(s) Q_{n+1}(s)+Q_{n-1}(s) Q_{n+1}^{(0)}(s)\right\}\right] \\
& +2(n-1) !\left(\begin{array}{c}
\mu \\
\lambda
\end{array}\right)^{n-1} \sqrt{(\lambda+n \mu-s)^{2}-4 n \lambda \mu} .
\end{aligned}
$$

It is seen that $K_{n}(s)$ is a polynomial in $s$ of exact degree $2 n-1$, with a root at $x=0$, and that the polynomial part of $L_{n}(s)$ is of degree $2 \mathrm{n}-2$. The Stieltjes inversion formula

$$
\psi_{0}(x)=\frac{1}{\pi} \lim _{\eta \rightarrow 0+} \int_{-\varepsilon}^{x} \Im B_{0}(\xi+i \eta) d \xi
$$

gives $\psi_{0}$ at all of its points of continuity. The above formulas show that $\Im B(x+i \eta)$ converges uniformly to zero as $\eta \rightarrow 0^{+}$if $x$ is in any closed interval containing no zeros of $K_{n}(x)$ and disjoint from the interval $|\lambda+n \mu-x| \leqq \sqrt{4 n \lambda \mu}$. Consequently over the interval

$$
|\lambda+n \mu-x|<\sqrt{4 n \lambda \mu}
$$

the measure $\psi_{0}$ has a continuous density $\psi_{0}^{\prime}(x)$ given by

$$
\psi_{0}^{\prime}(x)=\frac{(n-1) !}{2 \pi \lambda^{2}}\left(\frac{\mu}{\lambda}\right)^{n-1} \frac{V 4 n \lambda \mu-(\lambda+n \mu-x)^{2}}{Q_{n}^{2}(x)-Q_{n-1}(x) Q_{n+1}(x)} .
$$


In addition $\psi^{\prime}$ may have discrete masses at some or all of the zeros of $K_{n}(x)$. This possibility is discussed in $\S 7$, and treated in detail in the appendix.

To determine the polynomials $Q_{k}(x)$ for $k \geqq n$, let $R_{k}(x)=Q_{n+k}(x)$, $k \geqq-1$. Then

$$
R_{0}(x)=Q_{n}(x), \quad R_{-1}(x)=Q_{n-1}(x)
$$

and

$$
-x R_{k}(x)=n \mu R_{k-1}(x)-(\lambda+n \mu) R_{k}(x)+\lambda R_{k+1}(x), \quad k \geqq 0,
$$

which is a recurrence formula in which the coefficients are independent of $k$. The $\left\{R_{k}(x)\right\}$ can be expressed in terms of the Chebycheff polynomials $\left\{T_{k}(x)\right\},\left\{U_{k}(x)\right\}$ which satisfy

$$
x P_{k}(x)=\frac{1}{2} P_{k-1}(x)+\frac{1}{2} P_{k+1}(x), \quad k \geqq 1,
$$

and

$$
T_{0}(x)=1, \quad T_{-1}(x)=x, \quad U_{0}(x)=1, \quad U_{-1}(x)=0 .
$$

In fact, since

$$
V_{k}(x)=\left(\begin{array}{c}
n \mu \\
\lambda
\end{array}\right)^{k / 2} T_{k}\left(\frac{\lambda+n \mu-x}{\sqrt{4 n \lambda \mu}}\right)
$$

and

$$
W_{k}(x)=\left(\frac{n \mu}{\lambda}\right)^{k / 2} U_{k}\left(\frac{\lambda+n \mu-x}{\sqrt{4 n \bar{\lambda} \mu}}\right)
$$

are solutions of (4.13) for which

$$
V_{0}(x)=1, \quad V_{-1}(x)=\frac{\lambda+n \mu-x}{2 n \mu}, \quad W_{0}(x)=1, \quad W_{-1}(x)=0,
$$

we have

$$
\begin{aligned}
R_{k}(x)= & \frac{2 n \mu}{\lambda+n \mu-x} Q_{n-1}(x) V_{k}(x) \\
& +\left[Q_{n}(x)-\frac{2 n \mu}{\lambda+n \mu-x} Q_{n-1}(x)\right] W_{k}(x) \\
= & Q_{n}(x) W_{k}(x)-\frac{n \mu}{\lambda} Q_{n-1}(x) W_{k-1}(x), \quad k \geqq 0 .
\end{aligned}
$$

Hence for $k \geqq 0$

$$
Q_{n+k}(x)=\left(\frac{n \mu}{\lambda}\right)^{k / 2}\left[Q_{n}(x) U_{k}\left(\frac{\lambda+n \mu-x}{\sqrt{4 n \lambda \mu}}\right)\right.
$$




$$
\left.-\sqrt{\frac{n \mu}{\lambda}} Q_{n-1}(x) U_{k-1}\left(\frac{\lambda+n \mu-x}{\sqrt{4} n \lambda \mu}\right)\right]
$$

where $U_{-1}(\xi)=0$ and

$$
U_{k}(\xi)=\frac{\sin (k+1) \theta}{\sin \theta}, \quad \xi=\cos \theta, \quad k \geqq 0 .
$$

The system of polynomials $\left\{Q_{k}(x)\right\}$ is completely determined by (4.2) and (4.17).

A similar argument shows that the associated polynomials $\left\{Q_{k}^{(0)}(x)\right\}$ satisfy

$$
\begin{aligned}
Q_{n+k}^{(0)}(x)=\left(\frac{n \mu}{\lambda}\right)^{k / 2} & {\left[Q_{n}^{(0)}(x) U_{k}\left(\frac{\lambda+n \mu-x}{\sqrt{4 \lambda \mu}}\right)\right.} \\
& \left.-\sqrt{\frac{n \mu}{\lambda}} Q_{n-1}^{(0)}(x) U_{k-1}\left(\frac{\lambda+n \mu-x}{\sqrt{4 \lambda \mu}}\right)\right]
\end{aligned}
$$

for $k \geqq 0$.

5. The spectral measure of the one server and two server processes.

For the case of one server

$$
\begin{gathered}
Q_{0}(x)=1, \quad Q_{1}(x)=\frac{\lambda-x}{\lambda}, \quad Q_{2}(x)=\frac{\lambda+\mu-x}{\lambda} \cdot \frac{\lambda-x}{\lambda}-\frac{\mu}{\lambda}, \\
Q_{0}^{(0)}(x)=0, \quad Q_{1}^{(0)}(x)=-\frac{1}{\lambda}, \quad Q_{2}^{(0)}(x)=-\frac{\lambda+\mu-x}{\lambda^{2}} .
\end{gathered}
$$

Using these values in (4.7), (4.8) gives

$$
B_{0}(s)=-\frac{2(\mu-\lambda+s)+2 \sqrt{(\lambda+\mu-s)^{2}-4 \lambda \mu}}{4 \mu s} .
$$

The only possible pole of $B_{0}(s)$ is at $s=0$, and

$$
\begin{aligned}
\lim _{s \rightarrow 0}-s B_{0}(s) & =\frac{1}{2 \mu}[(\mu-\lambda)+|\mu-\lambda|] \\
& = \begin{cases}0 \quad \text { if } \mu \leqq \lambda \\
\frac{\mu-\lambda}{\mu} & \text { if } \mu>\lambda .\end{cases}
\end{aligned}
$$

Thus the spectral measure $\psi$ has the continuous density

$$
\psi^{\prime}(x)=\frac{1}{2 \pi \mu} \frac{\sqrt{4 \lambda \mu-(\lambda+\mu-x)^{2}}}{x}
$$

on the interval $|\lambda+\mu-x|<\sqrt{4 \lambda \mu}$ and has in addition a mass of amount 
$\frac{\mu-\lambda}{\mu}$ located at $x=0$ if $\mu>\lambda$, but has no extra mass if $\mu \leqq \lambda$. The polynomials are given by

$$
\begin{aligned}
Q_{k+1}(x)=\left(\frac{\mu}{\lambda}\right)^{k / 2} & {\left[\frac{\lambda-x}{\lambda} U_{k}\left(\frac{\lambda+\mu-x}{\sqrt{4 \lambda \mu}}\right)\right.} \\
& \left.-\sqrt{\frac{\mu}{\lambda}} U_{k-1}\left(\frac{\lambda+\mu-x}{\sqrt{4 \lambda \mu}}\right)\right], \quad k \geqq 0 .
\end{aligned}
$$

The associated polynomials are given by

$$
Q_{k+1}^{(0)}(x)=-\frac{1}{\lambda}\left(\frac{\mu}{\lambda}\right)^{k / 2} U_{k}\left(\frac{\lambda+\mu-x}{\sqrt{4 \lambda \mu}}\right), \quad k \geqq 0,
$$

and using (4.5) the function $B_{1}(s)$ is

$$
B_{1}(s)=\frac{\lambda+\mu-s-V(\lambda+\mu-s)-4 \lambda \mu}{2 \lambda \mu} .
$$

Hence the spectral measure $\psi_{1}$ of the associated process consists simply of the continuous density

$$
\psi_{1}^{\prime}(x)=\frac{1}{2 \pi \lambda \mu} \sqrt{ } 4 \lambda \mu-(\lambda+\mu-x)^{2}, \quad|\lambda+\mu-x| \leqq \sqrt{4 \lambda \mu} .
$$

We now turn to the two server case. The polynomials $Q_{k}, Q_{k}^{(0)}$ are again given by (5.1) and (5.2) for $k=0,1,2$ and a straightforward computation gives

$$
\begin{aligned}
K_{2}(s) & =4 \mu s\left[\left(\frac{\lambda-s}{\lambda}\right)^{2}+\frac{\mu}{\lambda} \frac{\lambda-s}{\lambda}+\frac{\mu}{\lambda}\right] \\
& =\frac{4 \mu s}{\lambda^{2}}\left[s^{2}-(2 \lambda+\mu) s+\lambda(\lambda+2 \mu)\right], \\
L_{2}(s) & =\frac{2 \mu}{\lambda^{2}}\left[(\lambda-s)(2 \mu-\lambda-2 s)+\lambda \sqrt{ }(\lambda+2 \mu-s)^{2}-8 \lambda \mu\right],
\end{aligned}
$$

and hence

$$
B_{0}(s)=-\frac{(\lambda-s)(2 \mu-\lambda-2 s)+\lambda \sqrt{(\lambda+2 \mu-s)^{2}-8 \lambda \mu}}{2 s\left[s^{2}-(2 \lambda+\mu) s+\lambda(\lambda+2 \mu)\right]} .
$$

Consequently the spectral measure $\psi$ of the two server queue has the density

$$
\psi^{\prime}(x)=\frac{\lambda}{2 \pi} \frac{\sqrt{8 \lambda \mu-(\lambda+2 \mu-x)^{2}}}{x\left[x^{2}-(2 \lambda+\mu) x+\lambda(\lambda+2 \mu)\right]}
$$


on the interval $|\lambda+2 \mu-x| \leqq V 8 \lambda \mu$, and in addition may have jumps at one or more of the zeros of the denominator of (5.9). Considering first the zero at $s=0$, we find that

$$
\begin{array}{r}
\lim _{s \rightarrow 0}-s B_{0}(s)=\frac{(2 \mu-\lambda)+\sqrt{(2 \mu-\lambda)^{2}}}{2(2 \mu+\lambda)} \\
=\left\{\begin{array}{ccc}
\frac{2 \mu-\lambda}{2 \mu+\lambda} & \text { if } 2 \mu>\lambda \\
0 & \text { if } 2 \mu \leqq \lambda .
\end{array}\right.
\end{array}
$$

Hence $\psi$ has a jump of magnitude $\frac{2 \mu-\lambda}{2 \mu+\lambda}$ at $x=0$ if $2 \mu>\lambda$, but no jump at $x=0$ if $2 \mu \leqq \lambda$. The other zeros of the denominator are

$$
\begin{aligned}
& s_{1}=\frac{2 \lambda+\mu}{2}-\frac{1}{2} \sqrt{\mu(\mu-4 \lambda)}, \\
& s_{2}=\frac{2 \lambda+\mu}{2}+\frac{1}{2} \sqrt{\mu(\mu-4 \lambda)} .
\end{aligned}
$$

These two roots are non-real if $\mu<4 \lambda$. Since $B_{0}(s)$ has no non-real poles we assume $\mu \geqq 4 \lambda$. If $\mu=4 \lambda$ the denominator has a double zero at $s=3 \lambda$. A simple computation shows that in this case the numerator also has a double zero at $s=3 \lambda$, and hence no jump of $\psi$ is involved. If $\mu>4 \lambda$ the residue at $s_{2}$ is easily computed. In fact

$$
\begin{aligned}
\left(\lambda+2 \mu-s_{2}\right)^{2}-8 \lambda \mu & =\frac{1}{4}\left[10 \mu^{2}-6 \mu \sqrt{\mu(\mu-4 \lambda)}-36 \lambda \mu\right] \\
& =\left[\frac{\mu-3 \sqrt{\mu(\mu-4 \lambda)}}{2}\right]^{2},
\end{aligned}
$$

and hence

$$
\begin{aligned}
& \left(\lambda-s_{2}\right)\left(2 \mu-\lambda-2 s_{2}\right)+\lambda \sqrt{\left(\lambda+2 \mu-s_{2}\right)^{2}-8 \lambda \mu} \\
& \quad=-\frac{\lambda}{2}\{\mu-3 \sqrt{\mu(\mu-4 \lambda)}-|\mu-3 \sqrt{\mu(\mu-4 \lambda)}|\} \\
& \quad=\left\{\begin{array}{cl}
0 & \text { if } \mu \geqq 3 \sqrt{\mu(\mu-4 \lambda)} \\
\lambda[3 \sqrt{\mu(\mu-4 \lambda)}-\mu] & \text { if } 3 \sqrt{\mu(\mu-4 \lambda)}>\mu .
\end{array}\right.
\end{aligned}
$$

The condition $3 \sqrt{ } \mu(\mu-4 \lambda)>\mu$ is equivalent to $\frac{\mu}{\lambda}>\frac{9}{2}$. Consequently $\psi$ has a jump at $x=s_{2}$ of magnitude

$$
\frac{\lambda[3 \sqrt{\mu(\mu-4 \lambda)}-\mu]}{\sqrt{\mu}(\mu-4 \lambda)[2 \lambda+\mu+\sqrt{\mu(\mu-4 \lambda)]}}
$$


if $\frac{\mu}{\lambda}>\frac{9}{2}$, but no jump there if $\frac{\mu}{\lambda} \leq \frac{9}{2}$. A similar calculation shows that $\psi$ never has a jump at $x=s_{1}$. If $\mu>4 \lambda$ then

$$
s_{2}<\lambda+2 \mu-\sqrt{8 \lambda \mu}
$$

except that equality holds when $\frac{\mu}{\lambda}=\frac{9}{2}$. The polynomials are given by

$$
\begin{aligned}
Q_{k+2}(x)= & \left(\frac{2 \mu}{\lambda}\right)^{k / 2}\left[\begin{array}{c}
(\lambda+\mu-x)(\lambda-x) \\
\lambda^{2}
\end{array} U_{k}\left(\frac{\lambda+2 \mu-x}{V 8 \lambda \mu}\right)\right. \\
& \left.-\sqrt{\frac{2 \mu}{\lambda}} \cdot \frac{\lambda-x}{\lambda} U_{k-1}\left(\begin{array}{c}
\lambda+2 \mu-x \\
V 8 \lambda \mu
\end{array}\right)\right]
\end{aligned}
$$

for $k \geqq 0$.

6. Probability distributions of various random quantities Associated with the one and two server processes.

In this section we compute the distributions of some interesting random variables connected with the one and two server processes. The transition probability function of the one server process is

$$
\begin{aligned}
P_{i, j}(t)= & Y\left(\frac{\mu}{\lambda}\right)\left(\frac{\lambda}{\mu}\right)^{j}\left(\begin{array}{c}
\mu-\lambda \\
\mu
\end{array}\right) \\
& +\left(\frac{\lambda}{\mu}\right)^{j} \int_{\lambda+\mu-\sqrt{4} \lambda \mu}^{\lambda+\mu+\sqrt{4} \lambda \mu} e^{-x t} Q_{i}(x) Q_{j}(x) \frac{\sqrt{4 \lambda \mu-(\lambda+\mu-x)^{2}}}{2 \pi \mu x} d x
\end{aligned}
$$

where $Y(z)$ is 0 if $z \leqq 1,1$ if $z>1$, and the polynomials are given by (5.5). The explicit expression for the distribution of waiting time, $W(t, \xi)$, of a customer arriving at time $t$ in the case of the one server queue may be readily derived from the integral representation (6.1). This is accomplished as follows: If at time $t$ the length of line (state of the process) consists of $n$ people with $n \geqq 1$ then the density of the waiting time of a person arriving at the moment $t$ is the gamma density of order $n$ whose scale parameter is $\mu$. The probability that at time $t$ the length of line is $n$ where initially the state of the process was $i$ is given by $P_{i n}(t)$. Consequently, for $\xi>0$

$$
d_{\xi} W_{i}(t, \xi)=\sum_{n=1}^{\infty} P_{i, n}(t) \frac{\mu^{n} \xi^{n-1} e^{-\mu \xi}}{(n-1) !} d \xi .
$$

Inserting the detailed formula (6.1) into the summation of (6.2) and performing the calculation, we obtain the formula

$$
d W_{i}(t, \xi)=\frac{\lambda}{\mu}(\mu-\lambda) e^{-(\mu-\lambda) \xi} d \xi+
$$




$$
+e^{-\mu \xi} \sqrt{\frac{\lambda}{\mu} \frac{1}{\pi}} \int_{\lambda+\mu-\sqrt{ } 4 \lambda \mu}^{\lambda+\mu+\sqrt{ } \lambda \lambda \mu} e^{-x t} Q_{i}(x) \Im\left[\left\{(\lambda-x) e^{i \theta}-\sqrt{\lambda \mu}\right\} e^{\xi \sqrt{\lambda \mu} e^{i \theta}}\right] d x d \xi
$$

where $\cos \theta=\frac{\lambda+\mu-x}{V 4 \lambda \mu}$ and $\Im$ stands for the imaginary part. We have tacitly assumed that $\frac{\mu}{\lambda}>1$ which of course is the interesting and practical case.

The evaluation of the sum is direct once it is realized that $Q_{n}(x)$ can be expressed according to (5.5) in terms of Chebycheff polynomials.

$$
Q_{n}(x)=\left(\frac{\mu}{\lambda}\right)^{\frac{n-1}{2}}\left[\left(\begin{array}{c}
\lambda-x \\
x
\end{array}\right) U_{n-1}\left(\begin{array}{c}
\lambda+\mu-x \\
V 4 \lambda \mu
\end{array}\right)-\sqrt{\gamma_{\lambda}^{\mu}} U_{n-2}\left(\begin{array}{c}
\lambda+\mu-x \\
V 4 \lambda \mu
\end{array}\right)\right], \quad n \geqq 1
$$

and

$$
U_{k}(z)=\frac{\sin (k+1) \theta}{\sin \theta}=\frac{\mathscr{F}\left(e^{i(k+1) \theta}\right)}{\sin \theta} \text { where } \cos \theta=z .
$$

Of course $P_{00}(t)$ evaluates the probability that a person arriving at time $t$ doesn't have to wait for his service to begin.

For computational purposes it might be remarked that the integrals of (6.1) and (6.3) may be expressed in terms of combinations of Bessel functions with imaginary arguments. This follows from the familiar fact that the Laplace transform of $\sqrt{1-t^{2}}$ for $-1 \leqq t \leqq 1$ involves Bessel functions [9]. This indeed is true of the majority of formulas connected with queueing. However, from the point of view of an understanding of the theory, and also for many practical purposes, we prefer the answer in the form of the integral representation.

The integral representation also enables us to determine directly the rate of approach to equilibrium in the ergodic case. The conclusion is immediate from relation (6.1) which implies the inequality

$$
\left|P_{i j}(t)-\left(\frac{\mu-\lambda}{\mu}\right)\left(\frac{\lambda}{\mu}\right)^{y}\right| \leqq M e^{-t(\lambda+\mu-\sqrt{ } 4 \lambda \mu)} .
$$

The asymptotic behavior of $P_{i j}(t)$ for large $t$ is easily obtained from formula (6.1). For example, for the case when $\lambda=\mu$

$$
P_{00}(t)=\frac{1}{2 \pi \mu} \int_{0}^{4 \lambda} e^{-x t} \sqrt{\frac{4 \lambda-x}{x}} d x,
$$

and when $t$ is large the main contribution to this integral is from the immediate neighborhood of $x=0$. In fact

$$
P_{00}(t) \sim \frac{1}{\pi \sqrt{ } \mu} \int_{0}^{1} \frac{e^{-x t}}{\sqrt{ } x} d x=\frac{1}{\pi V t \mu} \int_{0}^{t} \frac{e^{-\xi} d \xi}{\sqrt{ } \xi}
$$


and hence

$$
P_{00}(t) \sim \frac{1}{\sqrt{ } \pi \mu t} \text { as } t \rightarrow \infty .
$$

The cases when $\lambda>\mu$ or $\lambda<\mu$ can be dealt with in a similar way.

Now consider the distribution of the length of a busy period, or what is the same thing, the distribution of the first passage time from state 1 to state 0 , or what is the same again, the distribution of the time of absorption into the zero state for the related process $(\S 2)$, given that the related process starts in state 1 . If $\tilde{P}_{i j}(t)$ is the transition probability function of the related process, and $\psi_{1}$ is its spectral measure, then the probability $F_{10}(t)$ that absorption occurs before time $t$ is

$$
F_{10}(t)=\mu \int_{0}^{t} \tilde{P}_{11}(\tau) d \tau=\mu \int_{0}^{\infty} \frac{1-e^{-x t}}{x} d \psi_{1}(x)
$$

The Stieltjes transform of $\psi_{1}$ is given by (4.5) with $n=1$, and hence $\psi_{1}$ consists of the density

$$
\psi_{1}^{\prime}(x)=\frac{1}{2 \pi \lambda \mu} \sqrt{ } 4 \lambda \mu-(\lambda+\mu-x)^{2}
$$

on the interval $|\lambda+\mu-x| \leqq \sqrt{4 \lambda \mu}$. Consequently

$$
F_{10}(t)=\frac{1}{2 \pi \lambda} \int_{\lambda+\mu-\sqrt{\mathrm{d} \lambda \mu}}^{\lambda+\mu+\sqrt{4 \lambda \mu}} \frac{1-e^{-x t}}{x} \sqrt{4} \lambda \mu-(\lambda+\mu-x)^{2} d x
$$

is the probability that the length of a busy period for the one server queue is $\leqq t$. In a similar way the probability $F_{k 0}(t)$ that the queue will become idle before time $t$ when there are $k$ customers at time zero can be computed. Using the fact that the associated polynomials are given by

$$
Q_{n+1}^{(0)}(x)=-\frac{1}{\lambda}\left(\frac{\mu}{\lambda}\right)^{n / 2} U_{n}\left(\frac{\lambda+\mu-x}{\sqrt{ } 4 \lambda \mu}\right)
$$

one obtains

$$
\begin{aligned}
F_{k 0}(t)= & -\frac{1}{2 \pi \lambda} \int_{\lambda+\mu-\sqrt{4 \lambda \mu}}^{\lambda+\mu+\sqrt{4 \lambda \mu}} \frac{1-e^{-x t}}{x} \\
& \cdot\left(\frac{\mu}{\lambda}\right)^{\frac{k-1}{2}} U_{k-1}\left(\frac{\lambda+\mu-x}{\sqrt{4 \lambda \mu}}\right) \sqrt{\lambda \mu} \mu \mu-(\lambda+\mu-x)^{2} d x .
\end{aligned}
$$

It is also possible to compute the distribution of the number $N$ of customers arriving during a busy period, or more generally the number $N_{k}$ of customers arriving before the queue becomes idle given that initially there were $k$ customers in the queue. For this purpose we consider the random walk whose possible states are the integers $1,2,3, \cdots$ and an 
ignored absorbing state at 0 . The one step transition probabilities of the random walk are

$$
P_{i j}=\left\{\begin{array}{lll}
p_{i} & \text { if } \quad j=i+1, \\
q_{i} & \text { if } \quad j=i-1, \\
0 & \text { if } \quad j=i \text { or }|j-i|>1,
\end{array}\right.
$$

where

$$
p_{i}=\frac{\lambda_{i}}{\lambda_{i}+\mu_{i}}=\frac{\lambda}{\lambda+\mu}, \quad q_{i}=\frac{\mu_{i}}{\lambda_{i}+\mu_{i}}=\frac{\mu}{\lambda+\mu} .
$$

These quantities are independent of $i$ and we denote then by $p, q$. When the particle executing the random walk is in state 1 and a transition occurs, the particle goes to state 2 with probability $p$ and is absorbed into the zero state with probability $q$.

Each sample function of the associated queueing process generates in an obvious way a sample function of the random walk process, and it is clear that the random variable $N_{k}$, which is the number of customers arriving before the queue becomes idle, is the same as the total number of steps to the right made by the random walk before absorption at zero occurs. The total number of transitions of the random walk process which occur before absorption is a random variable $M_{k}$ related to $N_{k}$ and the initial state $k$ in such a way that

$$
M_{k}=k+2 N_{k} .
$$

If $P_{i j}^{n}$ denotes the $n$ step transition probability of the random walk, then

$$
\operatorname{Pr}\left\{M_{k}=m\right\}=q P_{k, 1}^{m-1}
$$

and hence

$$
\begin{aligned}
\operatorname{Pr}\left\{N_{k}=n\right\} & =\operatorname{Pr}\left\{M_{k}=k+2 n\right\} \\
& =q P_{k, 1}^{2 n+k-1} .
\end{aligned}
$$

Thus the distribution of $N_{k}$ is known if $P_{i j}^{n}$ is known.

An integral representation for $P_{i j}^{n}$ is obtained as follows. The random walk determines a system of polynomials by means of the recursion relations

$$
\left\{\begin{array}{l}
R_{1}(x)=1, \quad R_{0}(x)=0 \\
x R_{n}(x)=q R_{n-1}(x)+p R_{n+1}(x), \quad n \geqq 1 .
\end{array}\right.
$$

It is seen that $R_{n}(x)$ is a polynomial in $x$ of exact degree $n$. It can be shown that the polynomials $R_{n}(x)$ are orthogonal on $-1 \leqq x \leqq 1$ with respect to a uniquely determined measure $\alpha$ of total mass 1 . A proof of this fact which covers not merely the queueing case, but also the 
random walk arising in a similar way from a general birth and death process, is outlined in [5]. It is rather obvious that when $x^{n} R_{i}(x)$ is written as a linear combination of the polynomials $\left\{R_{k}(x)\right\}$ the coefficient of $R_{j}(x)$ is $P_{i j}^{n}$. Since it can be shown from the recurrence formulas that

$$
\int_{-1}^{1} R_{n}^{2}(x) d \alpha(x)=\frac{1}{\pi_{n}^{*}}
$$

where $\pi_{n}^{*}=\left(\frac{p}{q}\right)^{n-1}$, it follows that

$$
P_{i j}^{n}=\pi_{j}^{*} \int_{-1}^{1} x^{n} R_{i}(x) R_{j}(x) d \alpha(x),
$$

which is the desired representation of $P_{i j}^{n}$. Combining (6.9) and (6.11) we get an expression for the probability distribution of $N_{k}$ in terms of the measure $\alpha$. In particular the distribution of $N=N_{1}$, the number of customers arriving during a busy period, is

$$
\operatorname{Pr}\{N=n\}=q \int_{-1}^{1} x^{2 n} d \alpha(x) .
$$

The polynomials which satisfy the recurrence relation (6.10) are easily found to be

$$
R_{n}(x)=\left(\frac{\mu}{\lambda}\right)^{\frac{n-1}{2}} U_{n-1}\left(\frac{(\lambda+\mu) x}{\sqrt{ } 4 \lambda \mu}\right)
$$

from which it follows that the measure $\alpha$ consists of the density

$$
\alpha^{\prime}(x)=\frac{2}{\pi \sqrt{ } 4 \lambda \mu} \sqrt{1-\left(\frac{\lambda+\mu}{\sqrt{ } 4 \lambda \mu} x\right)^{2}}
$$

on the interval $|x| \leqq \frac{\sqrt{ } 4 \lambda \mu}{\lambda+\mu}$. Consequently

$$
\begin{aligned}
\operatorname{Pr}\{N=n\} & =\frac{\mu}{\lambda+\mu} \cdot \frac{2}{\pi} \cdot \frac{\lambda+\mu}{\sqrt{4 \lambda \mu}} \int_{-\frac{\sqrt{4} \lambda \mu}{\lambda+\mu}}^{\sqrt{4 \lambda \mu}} x^{2 n} \sqrt{1-\overline{\left(\frac{\lambda+\mu}{V} x\right)^{2}}} d x \\
& =\frac{\mu}{\lambda+\mu} \cdot\left(\frac{\sqrt{4 \lambda \mu}}{\lambda+\mu}\right)^{2 n} \cdot \frac{2}{\pi} \int_{-1}^{1} \xi^{2 n} \sqrt{1-\xi^{2}} d \xi
\end{aligned}
$$

We now turn to the two server queue. The transition probability function is

$$
\begin{aligned}
& P_{i j}(t)=Y\left(\frac{2 \mu}{\lambda}\right) \cdot \frac{2 \mu-\lambda}{2 \mu+\lambda} \cdot 2 \cdot\left(\frac{\lambda}{2 \mu}\right)^{j} \\
& +Y\left(\begin{array}{c}
2 \mu \\
9 \lambda
\end{array}\right)_{V \mu(\mu-4 \lambda)[2 \lambda+\mu+V \mu(\mu-4 \lambda)]}^{\lambda[3 V \mu(\mu-4 \lambda)-\mu]} \cdot 2 \cdot\left(\begin{array}{c}
\lambda \\
2 \mu
\end{array}\right)^{j} e^{-s_{2} t} Q_{i}\left(s_{2}\right) Q_{j}\left(s_{2}\right)+
\end{aligned}
$$




$$
+2\left(\frac{\lambda}{2 \mu}\right)^{j} \frac{\lambda}{2 \pi} \int_{\lambda+2 \mu-\sqrt{8 \lambda \lambda \mu}}^{\lambda+2 \mu+\sqrt{8 \lambda \mu}} e^{-x t} Q_{i}(x) Q_{j}(x) \frac{\sqrt{ } 8 \lambda \mu-(\lambda+2 \bar{\mu}-x)^{2}}{x\left[x^{2}-(2 \lambda+\mu) x+\lambda(\lambda+2 \mu)\right]} d x
$$

(unless $j=0$ in which case $2\left(\begin{array}{c}\lambda \\ 2 \mu\end{array}\right)$ is to be replaced by 1 ), where $Y(z)$ is 1 if $z>1,0$ if $z \leqq 1$, and

$$
s_{2}=\frac{2 \lambda+\mu}{2}+\frac{1}{2} V \mu(\mu-4 \lambda),
$$

and where the polynomials $Q_{i}(x)$ are given by (5.11). Once again the asymptotic behavior of $P_{i j}(t)$ for large $t$ is clearly exhibited by (6.16). In fact the first term on the right is either zero or else the largest term, and the second term, if not zero, is the second largest term. Finally the asymptotic behavior of the third term is a simple matter to investigate. For example if $\lambda=2 \mu$ it is found that

$$
P_{00}(t) \sim \frac{\lambda}{4} \frac{1}{\sqrt{\pi t}} \cdot
$$

By arguments entirely analogous to those used in the derivation of (6.3), we may obtain the form of the distribution of waiting time for a customer arriving at time $t$ in the two server queue. In fact, if $W_{i}(t, \xi)$ represents the cumulative distribution of waiting time for a person arriving at time $t$ where at time zero the state of the process was $i$, then

$$
d W_{i}(t, \xi)=\sum_{n=2}^{\infty} P_{i, n}(t) \frac{(2 \mu)^{n-1} \xi^{n-2}}{(n-2) !} e^{-2 \xi} d \xi, \quad \xi>0
$$

with $P_{i, n}(t)$ given by (6.16). We restrict attention only to the ergodic case when $2 \mu>\lambda$. Use of (6.16) in conjunction with (5.11) establishes the ultimate formula

(6.18) $d W_{i}(t, \xi) d \xi=\frac{2 \mu-\lambda}{2 \mu+\lambda} \cdot \frac{\lambda^{2}}{\mu} e^{-(2 \mu-\lambda) \xi}$

$$
\begin{aligned}
& +Y\left(\begin{array}{c}
2 \mu \\
9 \lambda
\end{array}\right) \frac{2 \lambda^{3}}{\mu} \frac{Q_{i}\left(s_{2}\right) e^{-s_{2} t} \sqrt{8 \lambda \mu} e^{-2 \mu \xi}}{\sqrt{\mu(\mu-4 \lambda[2 \lambda+\mu+\sqrt{\mu}(\mu-4 \lambda)]}} \\
& \text { - } \Im\left\{\left[\frac{\left(\lambda+\mu-s_{2}\right)\left(\lambda-s_{2}\right) e^{i \theta *}}{\lambda^{2}}-\sqrt{\frac{2 \mu}{\lambda}}\left(\frac{\lambda-s_{2}}{\lambda}\right)\right] e^{\xi \sqrt{2 \mu \lambda} e^{i \theta *}}\right\} \\
& +e^{-2 \mu \xi} \frac{\lambda^{3}}{2 \mu \pi} \sqrt{8 \lambda \mu} \int_{\lambda+2 \mu-\sqrt{8 \lambda \mu}}^{\lambda+2 \mu+\sqrt{8 \lambda \mu}} \frac{e^{-x t} Q_{i}(x)}{x\left[x^{2}-(2 \lambda+\mu) x+\lambda(\lambda+2 \mu)\right]} \\
& \text { - } \Im\left\{\left[\frac{(\lambda+\mu-x)(\lambda-x)}{\lambda^{2}} e^{i \theta}-\sqrt{\frac{2 \mu}{\lambda}}\left(\frac{\lambda-x}{\lambda}\right)\right] e^{\xi \sqrt{2 \mu \lambda} e^{i \theta}}\right\} d x
\end{aligned}
$$


where $\cos \theta^{*}=\frac{\lambda+2 \mu-s_{2}}{\sqrt{ } 8 \lambda \mu}$ and $\cos \theta=\frac{\lambda+2 \mu-x}{\sqrt{ } 8 \lambda \mu}$.

A busy period of the two server queue can now mean either a time interval during which both servers are busy, or else a time interval during which at least one server is busy.

Considering first the busy period for both servers, suppose the process is initially in state 2 , and let $T$ be the time at which the process first reaches state 1 . Then $\operatorname{Pr}\{T<t\}$ is the probability of absorption before time $t$ for the second associated process. Now the second associated process is similar to the first associated process of a one server queue in which the parameter $\mu$ has been replaced by $2 \mu$. Hence using (6.4),

$$
\operatorname{Pr}\{T<t\}=\frac{1}{2 \pi \lambda} \int_{\lambda+2 \mu-\sqrt{8} \bar{\lambda} \bar{\mu}}^{\lambda+2 \mu+\sqrt{8 \lambda \mu}} \frac{1-e^{-x t}}{x} \sqrt{ }-\sqrt{2} \mu-(\lambda+2 \mu-x)^{2} d x
$$

The distribution of the time before state 1 is reached when the initial state is $k(k \geqq 2)$ can be obtained from (6.6) in a similar way. By another argument of this kind it is seen that the distribution of the number $N$ of customers arriving during the busy period $T$ is obtained by replacing $\mu$ with $2 \mu$ in (6.15). Thus

$$
\operatorname{Pr}\{N=n\}=\frac{2 \mu}{\lambda+2 \mu}\left(\frac{\sqrt{ } 8 \lambda \bar{\mu}}{\lambda+2 \mu}\right)^{2 n} \int_{-1}^{1} \xi^{2 n} \sqrt{1-\xi^{2}} d \xi
$$

Next let us study the time during which at least one server is busy. Thus we suppose the initial state of the process is 1 and we denote by $T^{*}$ the time when the zero state is first reached. If $\psi_{1}$ is the spectral measure of the associated process, then by our previous argument

$$
\operatorname{Pr}\left\{T^{*}<t\right\}=\mu \int_{0}^{\infty} \frac{1-e^{-r t}}{x} d \psi_{1}(x) .
$$

Now the Stieltjes transforms $B_{1}(s)$ and $B_{2}(s)$ of the spectral measures of the first and second associated processes are related by

$$
B_{1}(s)=\frac{1}{\lambda+\mu-s-2 \lambda \mu B_{2}(s)}
$$

and from (4.5)

$$
B_{2}(s)=\frac{\lambda+2 \mu-s-\sqrt{(\lambda+2 \mu-s)^{2}-8 \lambda \mu}}{4 \lambda \mu} .
$$




$$
B_{1}(s)=\frac{1}{2 \mu} \cdot \frac{\lambda-s-\sqrt{ }(\lambda+2 \mu-s)^{2}-8 \lambda \mu}{s-(\mu-\lambda)}
$$

It follows that $\psi_{1}(x)$ has the continuous density

$$
\psi_{1}^{\prime}(x)=\frac{1}{2 \pi \mu} \cdot \frac{V 8 \lambda \mu-(\lambda+2 \mu-x)^{2}}{x-(\mu-\lambda)}
$$

on the interval $|\lambda+2 \mu-x| \leqq \sqrt{8 \lambda \mu}$, and in addition has a jump of magnitude $\frac{\mu-2 \lambda}{\mu}$ at $x=\mu-\lambda$ if $\mu>2 \lambda$, but has no jump if $\mu \leqq 2 \lambda$. Thus (6.21) becomes

$$
\begin{aligned}
& \operatorname{Pr}\left\{T^{*}<t\right\}= Y\left(\frac{\mu}{2 \lambda}\right)(\mu-2 \lambda) \frac{1-e^{-t(\mu-\lambda)}}{\mu-\lambda} \\
&+\frac{1}{2 \pi} \int_{\lambda+2 \mu-\sqrt{ } 8 \lambda \mu}^{\lambda+2 \mu+\sqrt{8 \lambda \mu}} 1-e^{-x t} \\
& x \frac{\sqrt{ } 8 \lambda \mu-(\lambda+2 \mu-x)^{2}}{-} \\
& x-(\mu-\lambda)
\end{aligned}
$$

where, as usual, $Y(z)=1$ if $z>1,0$ if $z \leqq 1$.

It is natural to next ask for the distribution of the number $N^{*}$ of customers arriving during the busy period $T^{*}$. This again leads to the study of a random walk on the integers $1,2, \cdots$ with an ignored absorbing state at 0 . The polynomials of the random walk satisfy the recursion relations

$$
\left\{\begin{array}{l}
R_{1}(x)=1, \quad R_{0}(x)=0 \\
x R_{1}(x)=p_{1} R_{2}(x) \\
x R_{n}(x)=q R_{n-1}(x)+p R_{n+1}(x), \quad n \geqq 2,
\end{array}\right.
$$

where

$$
p=\frac{\lambda}{\lambda+2 \mu}, \quad q=\frac{2 \mu}{\lambda+2 \mu}, \quad p_{1}=\frac{\lambda}{\lambda+\mu} .
$$

To compute the spectral measure $\alpha$ of this random walk we consider also the associated random walk obtained if the given one is stopped whenever state 1 is reached. Denoting the spectral measure of the associated random walk by $\beta$ we look for a relation between the Stieltjes transforms

$$
\int_{-1}^{1} \frac{d \alpha(x)}{x-z}, \quad \int_{-1}^{1} \frac{d \beta(x)}{x-z}
$$

analogous to the relation (2.5) for the spectral measures of a birth and death process and its associated process. Such a relation, applicable to a general random walk and its associated process, is proved in [6] and 
may be stated as follows. If the state space of the random walk is $0,1,2, \cdots$ and the one step transition probabilities are

$$
P_{i j}^{\prime}=\left\{\begin{array}{lll}
q_{i} & \text { if } & j=i-1, \\
r_{i} & \text { if } & j=i, \\
p_{i} & \text { if } & j=i+1, \\
0 & \text { if } & |j-i|>1,
\end{array}\right.
$$

where $p_{i}>0, q_{i}>0, r_{i} \geqq 0$, then the spectral measure $\alpha$ of the process and the spectral measure $\beta$ of the associated process are connected by the identity

$$
\int_{-1}^{1} \frac{d \alpha(x)}{x-s}=\frac{1 / p_{0}}{\frac{r_{0}-s}{p_{0}}-q_{1} \int_{-1}^{1} \frac{\mathrm{d} \beta(x)}{x-s}} .
$$

Now let $\alpha_{0}$ denote the spectral measure of the random walk determined by the polynomials (6.26); let $\alpha_{1}$ denote the spectral measure of the associated process and $\alpha_{2}$ the spectral measure of the second associated process. First applying (6.28) with $\alpha=\alpha_{0}, \beta=\alpha_{1}$, we get

$$
\int_{-1}^{1} \frac{d \alpha_{0}(x)}{x-s}=s_{-1}^{-1} \frac{d \alpha_{1}(x)}{x-s} .
$$

Then applying (6.28) with $\alpha=\alpha_{1}, \beta=\alpha_{2}$, we get

$$
\int_{-1}^{1} \frac{d \alpha_{1}(x)}{x-s}=\frac{-1}{s+p q \int_{-1}^{1} \frac{d \alpha_{2}(x)}{x-s}}
$$

But clearly $\alpha_{1}=\alpha_{2}$ so from (6.30)

$$
\int_{-1}^{1} \frac{d \alpha_{1}(x)}{x-s}=\frac{-s+\sqrt{s^{2}-4 p q}}{2 p q}
$$

where the radical must be determined by analytic continuation from positive values for $s>1$. Now (6.31) and (6.29) give

$$
\int_{-1}^{1} \frac{d \alpha_{0}(x)}{x-s}=\gamma \frac{(\gamma-1) s-\sqrt{s^{2}-4 p q}}{\left\{1-(\gamma-1)^{2}\right\} s^{2}-4 p q},
$$

where $\gamma=2 p / p_{1}=2(\lambda+\mu) /(\lambda+2 \mu)$ and $\gamma-1=\lambda /(\lambda+2 \mu)$ is positive and less than one. The Stieltjes inversion formula giving $\alpha_{0}$ at all of its points of continuity is

$$
\alpha_{0}(x)=\frac{1}{\pi} \lim _{\eta \rightarrow 0+} \int_{-1-\varepsilon}^{x} \Im\left[\int_{-1}^{1} \frac{d \alpha_{0}(y)}{y-\xi-i \eta}\right] d \xi
$$


and of course $\alpha$ has a jump at a point $x_{0}$ if and only if its Stieltjes transform has a pole there. A simple computation shows that the right side of (6.32) has no poles if $1 \leqq \gamma<2$, which is the case in our problem. Thus $\alpha_{0}$ consists of the continuous density

$$
\alpha_{0}^{\prime}(x)=\frac{\gamma}{\pi} \frac{\sqrt{4 p q-x^{2}}}{4 p q-\left\{1-(\gamma-1)^{2}\right\} x^{2}}
$$

on the interval $|x| \leqq \sqrt{4 p q}$, with $\gamma=2 p / p_{1}$. It is easy to express the probability distribution of $N^{*}$ in terms of $\alpha_{0}$, the result being

$$
\begin{aligned}
\operatorname{Pr}\left(N^{*}=n\right\} & =\frac{\mu}{\lambda+\mu} \int_{-\sqrt{4 p q}}^{\sqrt{4 p q}} x^{2 n} d \alpha_{0}(x) \\
& =\frac{2}{\pi} \frac{\mu}{\lambda+2 \mu}\left(\frac{\sqrt{8 \lambda \mu}}{\lambda+2 \mu}\right)^{2 n} \int_{-1}^{1} \xi^{2 n} \frac{\sqrt{1-\xi^{2}}}{1-\frac{4 \mu(\lambda+\mu)}{(\lambda+2 \mu)^{2}} \xi^{2}} d \xi .
\end{aligned}
$$

7. Results concerning the $n$ server queue. The method used in $\S 5$ to compute the spectral measures for the one and two server queues can be used in the same way to compute the spectral measure of a queue with three or more servers. Although the description of the spectral measure $\psi$ in terms of the parameters $\lambda, \mu$, and the number $n$ of servers becomes more and more complicated as $n$ increases, it is nevertheless possible to deduce certain general features of $\psi$. These general features are stated without proof in the next paragraph, and the proofs are supplied in the appendix.

The spectral measure $\psi$ of the $n$ server queue consists of a continuous density $\psi^{\prime}(x)$ on the interval

$$
\lambda+n \mu-\sqrt{4 n \lambda \mu}<x<\lambda+n \mu+\sqrt{4 n \lambda \mu},
$$

and in addition there may be a finite number of isolated jumps. The number of such isolated jumps is one of the integers $0,1,2, \cdots, n$ and these jumps all lie in the half-open interval

$$
0 \leqq x<\lambda+n \mu-\sqrt{4 n \lambda \mu} .
$$

If $n \mu>\lambda$ there is a jump at $x=0$ of magnitude $\rho$ given by

$$
\frac{1}{\rho}=\sum_{r=0}^{n-1} \frac{1}{r !}\left(\frac{\lambda}{\mu}\right)^{r}+\frac{1}{n !}\left(\frac{\lambda}{\mu}\right)^{n} \frac{n \mu}{n \mu-\lambda},
$$

but if $n \mu \leqq \lambda$ there is no jump at $x=0$. We form the polynomial

$$
F(\sqrt{b})=Q_{n}\left(\lambda(\sqrt{n \bar{b}}-1)^{2}\right)-\sqrt{n \bar{b}} Q_{n-1}\left(\lambda(\sqrt{n} \bar{b}-1)^{2}\right)
$$


which is of degree $2 n$ in $V b$. It has a zero of order $n$ at $\sqrt{b}=0$ and $n$ simple zeros $V b_{1}, \sqrt{b_{2}}, \cdots, \sqrt{ } b_{n}$ with $1=b_{1}<b_{2}<\cdots<b_{n}$. The spectral measure of the $n$ server queueing process has exactly $k$ jumps to the left of $x=\lambda+n \mu-\sqrt{4 n \lambda \mu}$ if and only if

$$
b_{k}<\frac{\mu}{2} \leqq b_{k+1}
$$

where we take $b_{0}=0$ and $b_{n+1}=+\infty$.

For the case $n=3$ the three critical values $b_{k}$ occur at $b=1 / 3$ and the two roots of $12 b^{2}-112 b+147=0$.

In discussing the busy period distributions for an $n$ server queue, one has to distinguish $n$ different cases. In the simplest case, one observes the time interval $T$ during which all $n$ servers are busy-that is, at time zero the process is in state $n$ and $T$ is the first time at which the process is in state $n-1$. The distribution of $T$ is of course obtained from (6.4) by replacing $\mu$ with $n \mu$, so that

$$
\operatorname{Pr}\{T \leqq t\}=\frac{1}{2 \pi \lambda} \int_{\lambda+n \mu-\gamma^{\overline{4} n \lambda \mu}}^{\lambda+n \mu+\sqrt{4 n \lambda \mu}} \frac{1-e^{-x t}}{x} \sqrt{4 n \lambda \mu-\left(\overline{\lambda+n \mu-x)^{2}}\right.} d x
$$

is the distribution of a busy period for all $n$ servers. Similarly, the distribution of the number $N$ of customers arriving during a busy period for all $n$ servers is obtained from (6.15):

$$
\operatorname{Pr}\{N=k\}=\frac{n \mu}{\lambda+n \mu}\left(\frac{\sqrt{4 n \lambda \mu}}{\lambda+n \mu}\right)^{2 k} \frac{2}{\pi} \int_{-1}^{1} \xi^{2 k} \sqrt{1-\overline{\xi^{2}}} d \xi .
$$

In the next simplest case one observes the time interval $T^{*}$ during which at least $n-1$ servers are busy-that is, at time zero the process is in state $n-1$ and $T^{*}$ is the first time at which the process is in state $n-2$. After a computation similar to that in $(6.21)-(6.25)$ we find

$$
\begin{aligned}
\operatorname{Pr}\left\{T^{*}<t\right\} & =Y\left(\frac{\mu}{n \lambda}\right)(\mu-n \lambda) \frac{1-e^{-t(n-1)(\mu-\lambda)}}{(n-1)(\mu-\lambda)} \\
& +\frac{1}{2 \pi} \int_{\lambda+n \mu-\sqrt{4 n \lambda \mu}}^{\lambda+n \mu+\sqrt{4 \lambda \lambda \mu}} \frac{1-e^{-x t}}{x} \cdot \frac{\sqrt{4 n \lambda \mu-(\lambda+n \mu-x)^{2}}}{x-(n-1)(\mu-\lambda)} d x
\end{aligned}
$$

where $Y(z)$ has its usual significance. If now $N^{*}$ is the number of customers arriving during a busy period for $(n-1)$ of the servers, then from (6.34) with

$$
p=\frac{\lambda}{\lambda+n \mu}, q=\frac{n \mu}{\lambda+n \mu}, \gamma=2 \frac{\lambda+(n-1) \mu}{\lambda+n \mu}
$$

we obtain 
(7.6) $\operatorname{Pr}\left\{N^{*}=k\right\}=\frac{2(n-1) \mu}{\pi}\left(\frac{\sqrt{4 n \lambda \mu}}{\lambda+n \mu}\right)^{2 k} \int_{-1}^{1} \xi^{2 k} \frac{\sqrt{ } 1-\xi^{2}}{1-\frac{4[\lambda+(n-1) \mu]_{\xi^{2}}}{(\lambda+n \mu)^{2}}} d \xi$.

Using the same kind of techniques it is possible to find the distribution of the length of a busy period for $m$ of the $n$ servers, and the distribution of the number of customers arriving during such a busy period.

8. Maximum length of the queue during a busy period.

Consider for the moment a general birth and death process with parameter $\lambda_{n}, \mu_{n}$ and with $\mu_{0}>0$. Suppose the initial state is $i$ and let $j>i$. It was shown in [5] that the probability that absorption at zero occurs without the state $j$ ever being visited is

$$
\begin{aligned}
{ }_{j} A_{i} & =\mu_{0} \int_{0}^{\infty} Q_{i}(x) \frac{d \psi^{(j)}(x)}{x} \\
& =\frac{\mu_{0} \sum_{k=i}^{j-1} \frac{1}{\lambda_{k} \pi_{k}}}{1+\mu_{0}^{j} \sum_{k=0}^{j-1} \frac{1}{\lambda_{k} \pi_{k}}}
\end{aligned}
$$

where $\psi^{(j)}$ is the spectral measure of the process on the states $0,1,2$, $\cdots, j-1$ which is obtained from the original process by stopping it whenever the $j$ state is reached.

We first use this result to compute the probability $\zeta_{n, j}$ that during a busy period for all servers in an $n$-server queue the maximum length of the queue is always less than $n+j$. This of course is just the probability that when the $n$th associated process starts out in its zero state, absorption occurs before it ever visits the $j$ th state, and hence (8.1) gives

$$
\zeta_{n, j}=\frac{n \mu}{\lambda} \cdot \frac{\left(\frac{n \mu}{\lambda}\right)^{j}-1}{\left(\frac{n \mu}{\lambda}\right)^{j+1}-1}
$$

A similar application of (8.1) to the $(n-1)$ st associated process gives the probability $\zeta_{n, j}^{*}$ that for the $n$ server queue during a busy period for at least $n-1$ of the servers the length of the queue is always less than $n-1+j$. The result is 


$$
\zeta_{n, j}^{*}=\frac{\frac{(n-1) \mu}{\lambda} \cdot \frac{\left(\frac{n \mu}{\lambda}\right)^{j}-1}{\frac{n \mu}{\lambda}-1}}{1+\frac{(n-1) \mu}{\lambda} \cdot \frac{\left(\frac{n \mu}{\lambda}\right)^{j}-1}{\left(\begin{array}{c}
n \mu \\
\lambda
\end{array}\right)-1}} \cdot
$$

Appendix A. The nature of the spectral measure for the $n$ server queue.

In this section we present the proofs of the statements made concerning the structure of the spectral measure for the $n$ server queue.

Let $\psi_{0}$ be the spectral measure of the $n$ server queue, $\psi_{k}$ be the spectral measure of the $k$ th associated process, and let $B_{k}(s)$ be the Stieltjes transform defined by (4.3). The relation between $B_{k}$ and $B_{k+1}$ is

$$
B_{k}(s)=\frac{1}{\lambda+k \mu-s-(k+1) \lambda \mu B_{k+1}(s)}
$$

and $B_{n}(s)$ is given by (4.5). In the interval $s_{0}=\lambda+n \mu-\sqrt{4 n \lambda \mu}<s<\lambda$ $+n \mu+\sqrt{4 n \lambda \mu}=s_{1}$ the imaginary part of $B_{n}(s+i \tau)$ converges to a positive limit as $\tau \rightarrow 0^{+}$. Consequently $\psi_{n}(x)$, and by induction each $\psi_{k}(x), 0 \leqq k \leqq n$, has a continuous spectrum in this interval. From (A.1) it is seen that $\psi_{k}$ has a jump at each point $x=s$ where the denominator $\lambda+k \mu-s$ $-(k+1) \lambda \mu B_{k+1}(s)$ has a simple zero. These jumps cannot occur in the interior of the interval of the continuous spectrum because there the imaginary part of the denominator is negative, and they cannot occur at the ends of the interval because there $B_{n}(s)$, and by induction each $B_{k}(s)$, has singularities which are not poles. From (4.5) it is found that $\psi_{n}$ has no jumps; in fact $B_{n}(s)$ increases steadily from zero at $s=-\infty$ to the value $(n \lambda \mu)^{-1 / 2}$ at $s=s_{0}$ and increases steadily from the value $-(n \lambda \mu)^{-1 / 2}$ at $s=s_{1}$ to zero at $s=+\infty$.

To locate the jumps, if any, of $\psi_{n-1}$, consider the places where the graph of the straight line $y=\lambda+(n-1) \mu-x$ intersects the graph of $y=n \lambda \mu B_{n}(x)$. No intersection occurs for $x>s_{1}$ because

$$
\lambda+(n-1) \mu-s_{1}-n \lambda \mu B_{n}\left(s_{1}\right)=-\mu-\sqrt{n \lambda \mu}<0 .
$$

Moreover, since

$$
\lambda+(n-1) \mu-s_{0}-n \lambda \mu B_{n}\left(s_{0}\right)=-\mu+\sqrt{n} \lambda \mu,
$$

and in view of the monotonicity of the two graphs, there is one intersection to the left of $x=s_{0}$ if $-\mu+\sqrt{n \lambda \mu}<0$, or equivalently if $\mu>n \lambda$, 
and no intersection if $\mu \leqq n \lambda$. Thus $\psi_{n-1}$ never has a jump to the right of the continuous spectrum and has one jump to the left of the continuous spectrum if $\mu>n \lambda$, no jump if $\mu \leqq n \lambda$. The jump, if $\mu>n \lambda$, is easily found to be at $x=(n-1)(\mu-\lambda)$.

It will be shown that none of the measures $\psi_{k}$ have any jumps to the right of the continuous spectrum. This has already been verified for $\psi_{n}$ and $\psi_{n-1}$, and we proceed by induction. Suppose it has been established for $k+1 \leqq r \leqq n-1$ that $\psi_{r}$ has no jumps to the right of $s_{1}$ and that $B_{r}\left(s_{1}\right)$ is finite, negative, and greater than $-(n \lambda \mu)^{-1 / 2}$. Since $B_{n-1}\left(s_{1}\right)=-\left(\mu+\sqrt{n \lambda \mu)^{-1}}\right.$, the inequality is certainly valid for $r=n-1$. From (A.1) we get

$$
B_{k}\left(s_{1}\right)=\frac{-1}{(n-k) \mu+2 \sqrt{n \lambda \mu}+(k+1) \lambda \mu B_{k+1}\left(s_{1}\right)},
$$

and by virtue of the assumed inequality for $r=k+1$ it follows that $B_{k}\left(s_{1}\right)$ is finite, negative, and greater than $-(n \lambda \mu)^{-1 / 2}$. Since $B_{k+1}(s)$ increases steadily from its finite negative value at $s=s_{1}$ to zero at $s=\infty$, it follows that the denominator of (A.1) is not zero for $s>s_{1}$ and $\psi_{k}$ has no jump to the right of $s_{1}$. This completes the induction.

Now suppose it has been established that for some $k, 1 \leqq k+1 \leqq n-1$, and some choice of $\lambda$ and $\mu$, the measure $\psi_{k+1}$ has exactly $r$ jumps. Let these jumps be at $x_{1}<x_{2}<\cdots<x_{r}$. Then $x_{r}<s_{0}$ and in each of the $r$ intervals $-\infty<s<x_{1}, x_{1}<s<x_{2}, \cdots, x_{r-1}<s<x_{r}$, the function $(k+1) \lambda \mu B_{k+1}$ increases steadily to $+\infty$, and thus in each interval its graph intersects the graph of $\lambda+k \mu-s$ exactly once. Consequently $\psi_{k}^{\prime}$ has exactly one jump in each of these intervals. In the interval $x_{r}<s<s_{0}$ the function $(k+1) \lambda \mu B_{k+1}$ increases steadily from $-\infty$ to its possibly finite value at $s_{0}$, and in this interval $\psi_{k}$ has either one or no jumps. Thus $\psi_{k}$ has at least $r$ and at most $r+1$ jumps. It follows that for any $\lambda, \mu$ the number of jumps of $\psi_{k}$ is at most $n-k$.

Setting $s=s_{0}$ in (A.1)

$$
B_{k}\left(s_{0}\right)=\frac{1}{2 \sqrt{n \lambda \mu-(n-k) \mu-(k+1) \lambda \mu B_{k+1}\left(s_{0}\right)}} .
$$

The necessary and sufficient condition that $\psi_{k}$ have one jump more than $\psi_{k+1}$ is that this expression be negative. Now it follows by induction starting from

$$
B_{n-1}\left(s_{0}\right)=\frac{1}{\sqrt{n \lambda \mu}-\mu}
$$

that for $k \leqq n-1$,

$$
B_{k}\left(s_{0}\right) \sim-\frac{1}{(n-k) \mu}
$$


as $\mu \rightarrow \infty$ with $\lambda$ fixed. Consequently, for any fixed $\lambda, \psi_{k}$ has exactly $n-k$ jumps for all sufficiently large $\mu$. On the other hand it follows by induction that for each $k \leqq n-1$,

$$
\lim _{\mu \rightarrow 0} \sqrt{\mu} B_{k}\left(s_{0}\right), \lambda \text { fixed, }
$$

exists and is positive but less than $(n \lambda)^{-1 / 2}$. Consequently, for any fixed $\lambda, \psi_{k}$ has no jumps for all sufficiently small $\mu$.

In order to make a more careful study of the number of jumps of $\psi_{k}$ we introduce the associated families of polynomials $\left\{Q_{m}^{(k)}(x)\right\}$ defined for $k=-1$ by

$$
Q_{m}^{(-1)}(x)=-\frac{1}{\lambda} Q_{m}(x)
$$

and for $k \geqq 0$ by the recursion formulas

$$
\begin{aligned}
Q_{r}^{(k)}(x) & =0 \quad \text { for } \quad r \leqq k, \\
Q_{k+1}^{(k)}(x) & =-\frac{1}{\lambda}, \\
-x Q_{r}^{(k)}(x) & =\mu_{r} Q_{r-1}^{(k)}(x)-\left(\lambda+\mu_{\imath}\right) Q_{r}^{(k)}(x)+\lambda Q_{r+1}^{(k)}(x), r \geqq k+1 .
\end{aligned}
$$

It is seen that except for the constant factor $-(1 / \lambda)$, the polynomials $Q_{m}^{(k)}(x)$ with $k$ fixed are the polynomials belonging to the $(k+1)$ th associated process and are orthogonal with respect to $\psi_{k+1}$. Applying (2.9) to the $k$ th associated process we obtain

$$
B_{k}(s)=\frac{1}{\lambda} \cdot \frac{n \mu Q_{n-1}^{(k)}(s) B_{n}(s)-Q_{n}^{(k)}(s)}{n \mu Q_{n-1}^{(k-1)}(s) B_{n}(s)-Q_{n}^{(k-1)}(s)} .
$$

In terms of the variable $b=\mu / \lambda$ we have $s_{0}=\lambda(1-\sqrt{ } n b)^{2}, n \mu B_{n}\left(s_{0}\right)=\sqrt{n b}$. If we let

$$
P_{r}^{(k)}(\sqrt{ } b)=\frac{-1}{\lambda} b^{-(r-k-1) / 2} Q_{r}^{(k)}\left(\lambda(1-\sqrt{n b})^{2}\right)
$$

then

$$
B_{k}\left(s_{0}\right)=\frac{1}{\lambda} \cdot \frac{P_{n}^{(k)}(\sqrt{ } b)-\sqrt{n} P_{n-1}^{(k)}(\sqrt{ } b)}{P_{n}^{(k-1)}(\sqrt{b})-\sqrt{n} P_{n-1}^{(k-1)}(\sqrt{ } b)} .
$$

The quantities $P_{r}^{(k)}(\xi)$ satisfy

$$
\left\{\begin{array}{l}
P_{k}^{(k)}(\xi)=0, P_{k+1}^{(k)}(\xi)=1, \\
-(n-r) \xi P_{r}^{(k)}(\xi)=r P_{r-1}^{(k)}(\xi)-2 \sqrt{n} P_{r}^{(k)}(\xi)+P_{r+1}^{(k)}(\xi),
\end{array}\right.
$$

for $k+1 \leqq r \leqq n$. By virtue of the form of this recurrence formula it follows that for each fixed $k$ the polynomials $P_{r}^{(k)}(\xi), k+1 \leqq r \leqq n$, form 
a finite system of orthogonal polynomials and that the polynomials $P_{r}^{(k+1)}(\xi), k+2 \leqq r \leqq n$, are the corresponding associated polynomials. Writing (A.4) in the form

$$
\begin{aligned}
P_{r+1}^{(k)}(\xi)-\sqrt{n} P_{r}^{(k)}(\xi)= & \sqrt{n}\left[P_{r}^{(k)}(\xi)-\sqrt{n} P_{r-1}^{(k)}(\xi)\right] \\
& +(n-r)\left[P_{r-1}^{(k)}(\xi)-\xi P_{r}^{(k)}(\xi)\right]
\end{aligned}
$$

it is easily shown by induction that all of the polynomials $P_{r}^{k}(\xi)$, $P_{r}^{(k)}(\xi)-\sqrt{n} P_{r-1}^{(k)}(\xi), k+1 \leqq r \leqq n$, are strictly positive for $\xi \leqq 0$.

Now the polynomial

$$
G_{n}^{(k-1)}(\xi)=P_{n}^{(k-1)}(\xi)-\sqrt{n} P_{n-1}^{(k-1)}(\xi)
$$

is a quasi-orthogonal polynomial, of exact degree $n-k$, belonging to the system of polynomials $P_{r}^{(k-1)}$, and the corresponding associated polynomial is $G_{n}^{(k)}(\xi)$. Consequently $G_{n}^{(k-1)}(\xi)$ has exactly $(n-k)$ distinct positive roots, say $\xi_{1}^{k-1}<\xi_{2}^{k-1}<\cdots<\xi_{n-k}^{k-1}$, and the $n-k-1$ roots of $G_{n}^{(k)}(\xi)$ lie one in each of the open intervals $\xi_{r}^{k-1}<\xi<\xi_{r+1}^{k-1}$.

The quantities $B_{k}\left(s_{0}\right)$ can be computed by using the recurrence formulas for the polynomials $P_{n}^{(k)}(\xi)$. In particular

$$
\begin{aligned}
B_{n-1}\left(s_{0}\right) & =\frac{-1}{\lambda \sqrt{ } b} \cdot \frac{1}{(2 \sqrt{ } n-\sqrt{ } b)-\sqrt{ } n} \\
& =\frac{-1}{\lambda \vee b(\sqrt{ } n-V b)}
\end{aligned}
$$

which checks with an earlier computation. Thus we see that the root $\xi_{1}^{n-2}$ of $G_{n}^{n-2}(\xi)$ is $\sqrt{n}$, and we already know that $\psi_{n-1}$ has no jump if $\sqrt{b} \leqq \xi_{1}^{n-2}$ and has one jump if $\sqrt{b}>\xi_{1}^{n-2}$. Suppose it has been established that $\psi_{k+1}$ has no jump if $V b^{-} \leqq \xi_{1}^{k}$, has $r$ jumps if $\xi_{r}^{k}<\sqrt{ } b \leqq \xi_{r+1}^{k}$ for $r=1,2, \cdots, n-k-2$, and has $n-k-1$ jumps if $V b>\xi_{n-k-1}^{k}$. This property is easily extended to $\psi_{k}$ by induction. In fact $\psi_{k}$ always has either the same number of jumps or else one more jump than $\psi_{k+1}$, and it has one more jump than $\psi_{k+1}$ if and only if the expression

$$
B_{k}\left(s_{0}\right)=\frac{1}{\lambda b^{1 / 2}} \frac{G_{n}^{(k)}(\sqrt{b})}{G_{n}^{(k-1)}(\sqrt{b})}
$$

is finite and negative. The result follows because of the interlacing of the roots of $G_{n}^{(k-1)}(\xi)$ and $G_{n}^{(k)}(\xi)$.

Summarizing, the number of jumps of $\psi_{k}$ to the left of the continuous spectrum is equal to the number of roots of $G_{n}^{(k-1)}(\xi)$ which are less than $V \vec{b}$. In particular the number of jumps of $\psi_{0}$, the spectral measure of the $n$ server process, is equal to the number of roots of the polynomial 


$$
\begin{aligned}
G_{n}^{(-1)}(\xi) & =P_{n}^{(-1)}(\xi)-\sqrt{n} P_{n-1}^{(-1)}(\xi) \\
& =\xi^{-n}\left[Q_{n}\left(\lambda(1-\xi \sqrt{n})^{2}\right)-\xi \sqrt{n} Q_{n-1}\left(\lambda(1-\xi \sqrt{n})^{2}\right)\right]
\end{aligned}
$$

which are less than $\sqrt{b}$.

Appendix B. The random walk polynomials derived from the infinitely many server queue.

In $\S 6$ we had occasion to consider, along with a birth and death process, the imbedded random walk. A system of polynomials, which are useful in a variety of problems, arises in this way from the infinitely many server process. These polynomials depend on a parameter $a=\lambda / \mu$ $>0$ and will be denoted by $r_{n}(x, a)$ or sometimes by $r_{n}(x)$. The purpose of this appendix is to list their useful properties.

The polynomials are defined by the recursion formulas

$$
\begin{aligned}
r_{0}(x, a) & =1, \\
x r_{0}(x, a) & =r_{1}(x, a), \\
(n+a) x r_{n}(x, a) & =n r_{n-1}(x, a)+a r_{n+1}(x, a), n \geqq 1 .
\end{aligned}
$$

They are orthogonal on the interval $-1 \leqq x \leqq 1$ with respect to a measure $\psi$ which consists entirely of jumps. If we let

$$
x_{k}=\sqrt{\frac{a}{k+a}}, k=0,1,2, \cdots
$$

then $\psi$ has equal jumps at $x_{k}$ and at $-x_{k}$ of magnitude

$$
\sigma_{k}=\frac{1}{2} \frac{a}{k+a} \frac{(k+a)^{k}}{k ! e^{k+a}}, k=0,1, \cdots
$$

and these have been normalized so the sum of all the jumps is one. The orthogonality relation is

$$
\sum_{k=0}^{\infty} r_{n}\left(x_{k}\right) r_{m}\left(x_{k}\right) \sigma_{k}+\sum_{k=0}^{\infty} r_{n}\left(-x_{k}\right) r_{m}\left(-x_{k}\right) \sigma_{k}=\frac{\delta_{n}, m}{\pi_{n}}
$$

where

$$
\pi_{n}=\frac{n+a}{a} \cdot \frac{a^{n}}{n !} .
$$

A generating function is

$$
\sum_{n=0}^{\infty} r_{n}(x, a) \frac{z^{n}}{n !}=e^{z / x}\left(1-\frac{x z}{a}\right)^{a\left(1-x^{2}\right) / x^{2}},
$$

and from this explicit representations of the polynomials can be obtained. For example 


$$
r_{n}(x, a)=\frac{1}{x^{n}} c_{n}\left(a \frac{1-x^{2}}{x^{2}}, \frac{a}{x^{2}}\right)
$$

where $c_{n}(x, a)$ are the Poisson-Charlier polynomials.

\section{REFERENCES}

1. N. T. J. Bailey, A continuous time treatment of a simple queue using generating functions, J. Roy. Stat. Soc., Ser. B 16 (1954), 288-291.

2. A. Erdélyi, et al, Higher transcendental functions, McGraw-Hill vol. II (1953).

3. W. Feller, An introduction to probability theory and its applications, Wiley (1950).

4. S. Karlin and J.McGregor, The differential equations of birth-and-death processes, and the Stieltjes moment problem, Trans. Amer. Math. Soc., 85 (1957), 489-546.

$5 . \quad \ldots$ - The classification of birth and death processes, Trans. Amer. Math. Soc., 86 (1957), 366-400.

6. __ Representation theory for random walks, to be published.

7. D. G. Kendall, Stochastic processes in the theory of queues. Ann. Math. Stat., 24 (1953) 333-354.

8. J. Kiefer and J. Wolfowitz, On the theory of queues with many servers, Trans. Amer. Math. Soc., 78 (1955), 1-18.

9. W. Lederman and G. E. H. Reuter, Spectral theory for the differential equations of simple birth-and-death processes, Phil. Trans. Roy. Soc., London-Ser. A vol. 246, No. 914 (1954), 321-369.

10. L. Takács, Investigation of waiting time problems by reduction to Markov processes, Acta Math. Acad. Sci. Hung., 6 (1955).

11. E. C. Titchmarsh, Theory of Fourier integrals, Oxford (1948).

12. D. V. Widder, The Laplace transform, Princeton (1941). 


\section{PACIFIC JOURNAL OF MATHEMATICS}

\section{EDITORS}

David Gilbarg

Stanford University

Stanford, California

R. A. Beaumont

University of Washington

Seattle 5 , Washington
A. L. Whiteman

University of Southern California Los Angeles 7, California

E. G. Straus

University of California

Los Angeles 24, California

\section{ASSOCIATE EDITORS}
E. F. BECKENBACH
C. E. BURGESS
M. HALL
E. HEWITT
A. HORN
V. GANAPATHY IYER
R. D. JAMES
M. S. KNEBELMAN

L. NACHBIN

I. NIVEN

T. G. OSTROM

H. L. ROYDEN
M. M. SCHIFFEI

G. SZEKERES

F. WOLF

K. YOSIDA

\section{SUPPORTING INSTITUTIONS}

UNIVERSITY OF BRITISH COLUMBIA

CALIFORNIA INSTITUTE OF TECHNOLOGY

UNIVERSITY OF CALIFORNIA

MONTANA STATE UNIVERSITY

UNIVERSITY OF NEVADA

OREGON STATE COLLEGE

UNIVERSITY OF OREGON

OSAKA UNIVERSITY

UNIVERSITY OF SOUTHERN CALIFORNIA
STANFORD UNIVERSITY

UNIVERSITY OF TOKYO

UNIVERSITY OF UTAH

WASHINGTON STATE COLLEGE

UNIVERSITY OF WASHINGTON

AMERICAN MATHEMATICAL SOCIETY

CALIFORNIA RESEARCH CORPORATION

HUGHES AIRCRAFT COMPANY

THE RAMO-WOOLDRIDGE CORPORATION

Printed in Japan by Kokusai Bunken Insatsusha

(International Academic Printing Co., Ltd.), Tokyo, Japan 


\section{Pacific Journal of Mathematics}

\section{Vol. 8, No. 1 \\ March, 1958}

Shimshon A. Amitsur, Commutative linear differential operators ......... 1

Masahiko Atsuji, Uniform continuity of continuous functions of metric

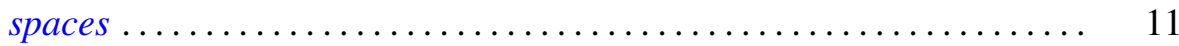

S. P. Avann, A numerical condition for modularity of a lattice . . . . . . . . . 17

Raymond G. D. Ayoub, A mean value theorem for quadratic fields........ 23

Errett Albert Bishop, Subalgebras of functions on a Riemann surface ..... . 29

Shaul Foguel, The relations between a spectral operator and its scalar

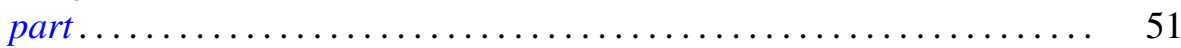

John Rolfe Isbell, Euclidean and weak uniformities ................. 67

Samuel Karlin and James L. McGregor, Many server queueing processes with Poisson input and exponential service times .............. 87

Paul Joseph Kelly and Ernst Gabor Straus, Curvature in Hilbert geometries....................................... 119

John W. Lamperti, Stationary measures for certain stochastic processes . . . 127

Richard Scott Pierce, Distributivity and the normal completion of Boolean

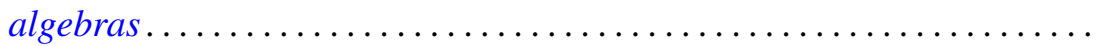

F. M. Ragab, Transcendental addition theorems for the hypergeometric

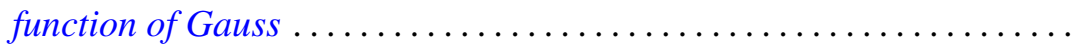

William T. Reid, Principal solutions of non-oscillatory self-adjoint linear differential systems ................................ 147

Maurice Sion, On general minimax theorems .................... 171

Chien Wenjen, On semi-normed ${ }^{*}$-algebras .................... 177 